\title{
Retrieval-based illusory recollections: Why study-test contextual changes impair source memory
}

\author{
Chad S. Dodson \\ University of Virginia, Charlottesville, Virginia
}

\begin{abstract}
Illusory source recollections can be manufactured purely by the conditions at retrieval. At encoding, individuals listened to words spoken by a male or a female voice and later, at retrieval, determined who initially had spoken the test word or whether it was a new word. In Experiment 1, individuals were instructed during the memory test to make a response of male or female only if they clearly recollected the speaker presenting the item during encoding and to respond don't remember otherwise. Presenting test words with the same voice (match condition) as the one that had presented the word at encoding, a different voice (mismatch condition), or no test voice had no effect on the rate of responding don't remember. Instead, matching and mismatching test voices created illusory recollections that were consistent with the test voice. Experiment 2 yielded similar results with a remember/know source test. In this article, a theory is proposed that explains the illusory recollection effects, and a multinomial model and procedure are used to separate and measure the contributions of source discrimination and illusory recollections to performance.
\end{abstract}

One of the more established principles of memory is the notion that memory performance is influenced by the degree of match between the properties of a memory trace and the features of a retrieval cue (e.g., J. R. Anderson, 1983; Bower, 1967; Hintzman, 1986; Johnson, Hashtroudi, \& Lindsay, 1993; McGeoch, 1942; Murnane, Phelps, \& Malmberg, 1999; Nairne, 2002; Raaijmakers \& Shiffrin, 1981; Schacter, 2001; Tulving, 1983). Perhaps the most famous study illustrating this phenomenon is Godden and Baddeley's (1975) demonstration that scuba divers recalled many more words when they were tested in the same context or environment as that in which they had learned the words rather than in a different one (e.g., study and tested under water versus study under water and tested on land). Many studies now have shown that a variety of different contextual changes between encoding and retrieval can affect recall and recognition performance (see Smith \& Vela, 2001, for a review). This notion that memory is context- or cue-dependent is further illustrated by the principles of encoding specificity, transfer-appropriate processing, and state-dependent memory (e.g., Morris, Bransford, \& Franks, 1977; Roediger, Weldon, \& Challis, 1989; Tulving \& Thomson, 1973). Remembering is likely to be successful when retrieval cues are uniquely connected to the desired memory (e.g., M. C. Anderson \& Neely, 1996; Nairne, 2002).

Remembering source information-specific information that identifies the origin of an event - is also affected by changes in the conditions between encoding and retrieval
(Dodson \& Shimamura, 2000; Leynes, Bink, Marsh, Allen, \& May, 2003; Palmeri, Goldinger, \& Pisoni, 1993; Roediger, McDermott, Pisoni, \& Gallo, 2004). Dodson and Shimamura presented participants with words that were spoken by a male and a female voice. Remembering who spoke a word earlier was much better when the test words were presented by the same voice as the one that had presented the word at encoding than when they were presented by a different voice. Likewise, Roediger et al. (2004) found that participants were strongly biased to respond that items had been presented at study by the voice that happened to be presenting the item at test, resulting in good performance when the study and test voices matched and poor performance when there was a mismatch (see Senkfor \& Van Petten, 1998, for similar results). Ochsner, Schacter, and Edwards (1997) observed similar findings in a related paradigm. They had participants encode faces with slightly positive or negative expressions while simultaneously hearing an angry or a happy voice. Although there was no relation between the tone of voice and the expression on the face at encoding, when shown a face at retrieval and asked to recall the tone of voice, participants were biased to respond that the tone of voice matched the expression on the face.

Why is memory performance influenced by the match or mismatch between the study and the test conditions? Mismatching test cues may activate other memories and, consequently, interfere with access to and retrieval of the desired memory. Indeed, in many paradigms, forgetting is often caused by processes, such as blocking or cue over-

C.S.Dodson, cdodson@virginia.edu 
load, that affect the accessibility of the desired memory (see M. C. Anderson \& Neely, 1996; Nairne, 2002; Wixted, 2004). This interference account was essentially what Dodson and Shimamura (2000) proposed to explain the effects of encoding specificity on source memory. They suggested that mismatching test cues increased the likelihood that participants failed to remember any diagnostic source information and, consequently, had to guess in making a response.

This article, however, investigates a different account of the cause of source attribution errors when retrieval cues mismatch the properties of the memory trace. Instead of guessing because of the failure to remember any source information, an alternative account focuses on the occurrence of illusory recollections. When a retrieval cue mismatches properties of the memory trace, such as hearing a female present a test item that was originally spoken by a male at encoding, individuals may experience an illusory recollection that is consistent with the retrieval cue, such as falsely recollecting that the female spoke the item at encoding. This hypothesis about retrieval-based illusory recollections builds on the ideas of Ochsner et al. (1997), as well as Tulving's (1983) theory of synergistic ecphory, whereby recollective experience is constructed from information activated from the memory trace and information in the retrieval cue. Moreover, the notion that recollective experience is a construction is fundamental to many prominent theories of memory, such as the source-monitoring framework (e.g., Johnson, Hashtroudi, \& Lindsay, 1993), the constructive memory framework (e.g., Schacter, Norman, \& Koutstaal, 1998), and fuzzy trace theory (e.g., Brainerd, Payne, Wright, \& Reyna, 2003), and is consistent with the thinking of other theorists (e.g., Higham \& Vokey, 2004; Lindsay \& Kelley, 1996; Whittlesea, 2004).

In addition, a variety of different paradigms document that illusory recollections play a central role in contributing to errors (e.g., Hannigan \& Reinitz, 2001; Lampinen, Meier, Arnal, \& Leding, 2005). In the eyewitness testimony paradigm, people sometimes claim to recollect having seen suggested items (e.g., Dodson \& Krueger, 2006; Frost, 2000; Lindsay, 1990; Zaragoza \& Lane, 1994). Similarly, people occasionally remember specific details about items that were never encountered before (e.g., that it was presented by a woman's voice) when the item is semantically related to studied items (e.g., Payne, Elie, Blackwell, \& Neuschatz, et al., 2004). Moreover, false and true memories are sometimes associated with similar kinds and amounts of brain activity, further supporting the idea that they can elicit comparable phenomenal experiences (e.g., Schacter \& Slotnick, 2004).

The present research extends this notion of illusory recollections to the question of why changes in studytest context affect memory performance. Specifically, I investigate the hypothesis that elements of the retrieval environment can manufacture illusory recollections.

\section{EXPERIMENT 1}

I used the following procedure to test the recollection failure and illusory recollection accounts. Participants were presented with words spoken by a male or a female. Source memory was assessed for test words presented only visually, with the same study voice, or with a mismatched voice. When responding to the test items, the participants initially made an old-new judgment. For items judged old, the participants then made a source judgment about who said it during the study phase or responded that they could not remember who had said it (i.e., Eric, Julie, or don't remember). The critical feature of this paradigm is that participants were directed to select Eric or Julie only if they vividly remembered this source presenting the item during the study phase-instructions that are similar to those used to guide participants to make a remember response in the remember/know paradigm (e.g., Gardiner \& Java, 1993; Tulving, 1985). If they did not have a vivid recollection, they were directed to choose don't remember. Above all, the participants were warned not to guess Eric or Julie. This procedure (or a similar version of it) has been used in many studies to increase the likelihood that source attributions are based on recollective experience and not on guesses (e.g., Gallo \& Roediger, 2003; Lampinen, Neuschatz, \& Payne, 1999; Macrae, Schloerscheidt, Bodenhausen, \& Milne, 2002; Payne et al., 1996; Roediger et al., 2004).

The recollection failure and illusory recollection accounts make opposite predictions about the effect of matching and mismatching test voice conditions on the rate of don't remember responses and incorrect source attributions (i.e., responding Eric to an item presented by Julie at encoding). The recollection failure account assumes that presentation of the wrong voice at test (i.e., mismatching test cues) blocks access to memory for the correct voice information. Consequently, it predicts an increase in don't remember responses in the mismatch condition, relative to the match condition. By contrast, the illusory recollection hypothesis assumes that presentation of the wrong voice at test actively misleads participants into falsely recollecting that the item was presented earlier by the same voice at encoding. These false recollections will cause participants to choose the incorrect source, rather than to respond don't remember. Therefore, the illusory recollection account predicts (1) no change in the rate of responding don't remember in the mismatch and match conditions and (2) an increase in incorrect source attributions in the mismatch condition than in the match condition.

\section{Method}

Participants. Thirty-five volunteers were recruited from the student population of the University of Virginia.

Design and Materials. The stimulus material consisted of 180 five- and six-letter words presented in the visual and auditory modes (i.e., spoken by a male or a female voice). An Apple Macintosh computer and the SoundEdit (1992) program was used to digitize and record the words. During the experiment, these words were presented at volumes corresponding to normal conversational levels.

The words were divided equally into three 60 -word sets that were matched for word frequency $(M=66$; Kučera \& Francis, 1982). Across participants, these sets were counterbalanced across three study conditions: (1) 60 study words spoken by a male, (2) 60 study words spoken by a female, and (3) 60 new words not presented at study. Thus, the study phase consisted of the presentation of 120 tar- 
Table 1

Percentages of Responses to Studied and New Words in the Male-Test-Voice, Female-Test-Voice, and No-Test-Voice Conditions in Experiment 1

\begin{tabular}{llrrrr}
\hline & & \multicolumn{4}{c}{ Response } \\
\cline { 3 - 6 } Test Voice & Source & Male & Female & $\begin{array}{c}\text { Don't } \\
\text { Remember }\end{array}$ & New \\
\hline Male & Male & $\mathbf{5 0 . 4}$ & 4.8 & 20.2 & 24.6 \\
& Female & 13.5 & $\mathbf{4 1 . 7}$ & 23.0 & 21.9 \\
& New & 1.7 & 1.3 & 4.3 & $\mathbf{9 2 . 8}$ \\
Female & Male & $\mathbf{4 2 . 9}$ & 12.2 & 22.2 & 22.6 \\
& Female & 5.4 & $\mathbf{5 4 . 7}$ & 23.7 & 16.3 \\
& New & 0.7 & 1.1 & 6.2 & $\mathbf{9 2 . 0}$ \\
None & Male & $\mathbf{4 1 . 0}$ & 9.5 & 24.5 & 25.1 \\
& Female & 8.7 & $\mathbf{4 3 . 8}$ & 25.4 & 22.1 \\
& New & 0.4 & 1.9 & 5.8 & $\mathbf{9 2 . 0}$ \\
\hline
\end{tabular}

Note-Bold numbers refer to the correct response.

get words, half spoken by a male voice and the other half spoken by a female voice. However, in order to improve overall memory, these target words were repeated three separate times, with the same voice presenting the word at each repetition. The words were presented in a random order, with the constraint that words spoken by the same voice would not occur consecutively more than three times. Six additional filler words were presented at the beginning of the list, and 6 filler words were presented at the end, to reduce primacy and recency effects. In addition to the auditory presentation of the words, the participants were also presented with the word visually on each trial.

Immediately following the study phase, I assessed memory for 90 old words and 45 new words. On the test, the type of presentation (i.e., visually only, male voice, or female voice) was crossed with the type of item (i.e., new words, male study words, or female study words). On a third of the trials, the test cue was simply the word presented visually on the monitor (no-test-voice condition). For another third of the trials, the participants heard the test word spoken by the female voice that had been used during the study phase, along with the visual presentation of the word (female test voice condition). For the remaining third of the test words, participants heard the test word spoken by the male voice that had been used during the study phase, along with the visual presentation of the word (male test voice condition). The words were presented in a random order, with the constraint that words from the same condition would not appear consecutively more than three times. In addition to these 135 test words, there were an additional 10 words at the beginning as a way to familiarize the participants with the testing procedure. These 10 words came from the buffer items presented at the beginning and end of the study list. Across participants, the specific words in the different conditions were fully counterbalanced. ${ }^{1}$

Procedure. In the study phase, the items were presented in an incidental and self-paced fashion. The participants were told that the purpose of the experiment was to study the ability to perceive and imagine voices. No mention was made of a later memory test. The participants were instructed that they would hear words spoken by a male (identified as Eric) and a female (identified as Julie) and, immediately after presentation, they were to imagine hearing the same person say the word again. The participants used the numbers on the keypad to rate on a 1-to-7 scale how easy it was to imagine the word being spoken by that voice $(1=$ very easy, $7=$ very difficult $)$. The next study item appeared immediately after each response. The first five words were practice trials in order to demonstrate the task to the participants.

After the study phase, the participants were given a surprise source memory test. They were told that the test would consist of both new words and old words that had been spoken by the two speakers and that they would use labeled keys on the keyboard to make their judgments. The participants were asked to determine whether a test word was old (i.e., it had been encountered before) or new. For words receiving a judgment of old, the participants then made a judgment about whether the word had been spoken by a male at study or a female at study or whether they could not remember (i.e., don't remember). I emphasized to the participants that they should make a response of Eric or Julie only if they vividly and clearly recollected the voice presenting the word during the study phase. Above all, I admonished the participants to not guess and to select don't remember if they could not distinctly remember who had spoken the word during the study phase. All test words appeared visually in the center of the screen with the response options of either old and new or Julie, Eric, and don't remember appearing $2 \mathrm{~cm}$ below the word. After each response, the screen cleared and was followed by a 1-sec delay before the presentation of the next word. The participants were instructed that the test words would appear in one of three ways: spoken by the male voice, spoken by the female voice, or not spoken (i.e., presented visually only). They were told, however, that the test voice would not necessarily correspond to the voice that had originally presented the word.

\section{Results and Discussion}

Table 1 presents memory performance across the three test conditions (male test voice, female test voice, or no test voice) for the three kinds of test items. All analyses of performance are based on $2 \times 3$ ANOVAs with study source (male or. female) and test voice condition (male, female, or no test voice) as within-subjects factors. I assessed item recognition with corrected recognition scores (i.e., by subtracting the false alarm rate to new items from the hit rate to studied items). There was a main effect of study source $\left[F(1,34)=6.91, M S_{\mathrm{e}}=0.01, p<.05\right]$, reflecting slightly higher recognition performance for items presented at study by the female $(72 \%)$ than for those presented by the male (68\%), and no other significant effects (all $F_{\mathrm{S}}<1.21$ ).

Source identification performance was measured by examining the percentage of items identified as old that were then attributed to the correct source (referred to as the conditional source identification score; Murnane \& Bayen, 1996). For example, the source score for the female study items was calculated with the following formula: $p$ ("female"|female) $/ p$ (“old"|female), where $p$ ("old"|female) refers to the probability of responding old to a word that had actually been spoken by the female in the study phase. In this experiment, chance with this measure was .33 , since there were three possible responses: (1) a correct source attribution, (2) an incorrect source attribution, or (3) a response of don't remember. Table 2 displays these conditional correct, incorrect, and don't re-

Table 2

Percentages of Recognized Items That Were Attributed to the Correct Source or the Incorrect Source or Whose Source Was Not Remembered in Experiment 1

\begin{tabular}{|c|c|c|c|c|c|c|}
\hline & \multicolumn{6}{|c|}{ Test Condition } \\
\hline & \multicolumn{2}{|c|}{ Match } & \multicolumn{2}{|c|}{ Mismatch } & \multicolumn{2}{|c|}{ No Test Voice } \\
\hline & $M$ & $\overline{S E M}$ & $M$ & $S E M$ & $M$ & SEM \\
\hline Correct attribution & 66.1 & .03 & 50.7 & .04 & 53.7 & .03 \\
\hline Incorrect attribution & 5.4 & .01 & 18.8 & .03 & 12.2 & .02 \\
\hline Don't remember & 28.4 & .03 & 30.0 & .03 & 34.2 & .04 \\
\hline
\end{tabular}

Note-Match and mismatch refer to conditions in which the voice saying the test item either matched or mismatched that of the speaker of the item at encoding. 
member responses in the study-test match, mismatch, and no-test-voice conditions.

The analysis of conditional correct source scores showed a main effect of test voice $\left[F(2,68)=3.75, M S_{\mathrm{e}}=\right.$ $0.015, p<.05]$, a significant study source $\times$ test voice interaction $\left[F(2,68)=14.20, M S_{\mathrm{e}}=0.031, p<.0001\right]$, and no other significant effects. Planned comparisons revealed that source identification was better in conditions when the test voice matched the study voice $(66 \%)$ than in conditions when either the study and the test voices mismatched $(51 \%)[t(34)=5.04, p<.001]$ or there was no test voice $(54 \%)[t(34)=5.20, p<.001]$. As can be seen in Table 2, there was no difference in performance between the mismatch and the no-test-voice conditions $[t(34)=1.25]$.

Turning to the central question of this experiment, are the differences in source identification performance between the match and the mismatch conditions caused by illusory recollections, by don't remember responses, or by a mixture of the two?

To answer this question, I analyzed illusory recollections by examining the rate at which the participants selected the incorrect voice as a response when they recognized the test item as old [e.g., $p$ ("male"|female) $/ p$ ("old"|female)]. As a reminder, I warned the participants not to guess but only to respond either male or female when they vividly remembered this speaker presenting the test item during the study phase. Therefore, these incorrect source attributions are presumably based on recollective experience. This ANOVA revealed a significant study source $\times$ test voice interaction $\left[F(2,68)=19.01, M S_{\mathrm{e}}=.017, p<\right.$ $.0001]$ and no other significant effects $(F \mathrm{~s}<1)$. Illusory recollections, as is shown in Table 2, were much more frequent when the test voice mismatched the study source (.19) than when there was a match (.05) $[t(34)=5.43$, $p<.0001]$ or when there was no test voice $(.12)[t(34)=$ $2.87, p<.01]$. Illusory recollections were less frequent in the match condition than in the no-test-voice condition $[t(35)=4.02, p<.001]$.

Finally, I analyzed the percentage of items judged old that were given a response of don't remember, as shown in the bottom row of Table 2 . This analysis yielded a marginally significant main effect of test voice $[F(2,68)=2.41$, $\left.M S_{\mathrm{e}}=0.021, p<.10\right]$ and no other significant effects $\left(F_{\mathrm{S}}<1\right)$. Responding don't remember about who spoke the item tended to be more frequent when there was no test voice $(34 \%)$ than when there was either a male or a female test voice (29\% for both). There was no effect of matching or mismatching test voices on the rate of responding don't remember $[t(34)=1.00, p>.32]$. This pattern of don't remember responses conflicts with the predictions of the recollection failure account. Instead of increasing don't remember responses, mismatching test voices decreased don't remember responses, relative to the no-test-voice condition (i.e., $30 \%$ vs. $34 \%$, respectively) $[t(34)=1.72, p<.10]$.

In summary, regardless of whether the test voice matched or mismatched who had actually spoken the item during the study phase, there was no difference in the rate at which the participants responded don't remember, since they used this response approximately $29 \%$ of the time in both con- ditions. Instead, matching and mismatching test voices affected how often the participants selected the incorrect speaker as a response - that is, the occurrence of illusory recollections. Incorrect source attributions were nearly four times as common in the mismatch condition (.19) as in the match condition (.05). These results are consistent with the predictions of the illusory recollection account and indicate that test voices create illusory recollections that are consistent with the test voice. Moreover, the absence of any effect of matching and mismatching test voices on the rate of responding don't remember is inconsistent with the recollection failure account. In short, mismatching test voices do not appear to block access to a desired memory; instead, they create illusory recollections.

\section{EXPERIMENT 2}

Experiment 1 shows that illusory recollections about who-said-what can be manufactured at the time of retrieval by having a voice present the test item. This conclusion, however, rests on the assumption that the participants used the don't remember response instead of guessing a source. Although the participants used this response nearly $30 \%$ of the time when they recognized an item as old, this does not prove that all guessing responses have been prevented from contributing to source attributions.

Therefore, Experiment 2 used a combined source memory and remember/know (R/K source) procedure to examine the influence of test voices on illusory recollections. Several studies have used the $\mathrm{R} / \mathrm{K}$ source procedure to examine variables that influence the degree to which source judgments are based either on recollection of specific details (i.e., a remember judgment) or on a sense of familiarity in the absence of memory for any specific source details (i.e., a know judgment; e.g., Dewhurst \& Hitch, 1999; Hicks, Marsh, \& Ritschel, 2002; Meiser \& Bröder, 2002; Perfect, Mayes, Downes, \& Van Eijk, 1996).

The $\mathrm{R} / \mathrm{K}$ source paradigm has several advantages over the don't remember paradigm used in Experiment 1. First, there is no need to infer that the absence of a don't remember response means that the source judgment is based on the presence of a recollective experience. The $\mathrm{R} / \mathrm{K}$ source paradigm bypasses this inference by requiring participants to make a deliberate assessment about the memorial basis for each response - that is, by providing a remember or know judgment for each source attribution. In addition, this paradigm allows for the measurement of the relative influence of test voices both on the occurrence of illusory recollections and on guessing processes when participants fail to recollect specific source information. That is, this paradigm allows for the construction of a multinomial model that can measure the separate contributions of illusory recollections and guessing biases.

During the past quarter century, Batchelder, Riefer, and colleagues' application of multinomial models to source memory research has been an important advance because these models are able to separate the influence of response biases and other variables from the measurement of source memory (e.g., Batchelder \& Riefer, 1990, 1999). However, multinomial models of source memory make very spe- 
cific assumptions about the cause of source identification errors, such as claiming that Julie said something that was actually spoken by Eric. These models assume that source identification errors are produced by guessing processes when participants fail to recollect sufficient diagnostic source information. In other words, these models assume that recollection failure causes all source identification errors. These models were not constructed to measure other possible causes of source errors, such as illusory recollections. Therefore, this article will present a method and a multinomial model that disentangle the contributions of recollection failure and illusory recollection to source identification performance.

In terms of the behavioral results, illusory recollections will be manifested by greater false remember responses in the mismatch than in the match condition. By contrast, the recollection failure account specifies no mechanism that would lead to an increase in false remember responses. However, the key predictions of this experiment involve the illusory recollection parameter of the model. If test voices create illusory recollections, this will increase the illusory recollection parameter of the model, as compared with the no-test-voice condition.

\section{Method}

Participants. Twenty-seven volunteers were recruited from the student population of the University of Virginia.

Design and Materials. The identical design and stimuli from Experiment 1 were used, except for the following change. There was just a single source memory test that consisted of 180 items (120 old and 60 new items), with one third of the test items presented by the male voice, one third presented by the female voice, and one third presented with no voice. All other aspects of the study and test phases were identical to those in Experiment 1.

Procedure. I used the same study instructions as those in Experiment 1 . For the memory test, the participants were asked to identify whether the word had been spoken during the test study phase or whether the test word was new, by responding with the keys on the keyboard corresponding to Julie, Eric, or new. However, following responses of Julie or Eric, the participants then made remember or know judgments as a basis for their source attribution (e.g., Hicks et al., 2002; Rajaram, 1993). However, the exact instructions given to the participants about making remember and know judgments were slightly different from those in previous studies. The participants were told to use a remember judgment when they recollected specific details about encountering the test item during the study phase. That is, I constrained this response from the typical instruction about the presence of a recollective state (regardless of what information is producing this state) to recollective states that are driven only by source-identifying information about who had spoken the item. This change was designed to minimize the contributions of noncriterial recollections - that is, non-source-specifying recollective information, such as remembering that the test item provoked an idiosyncratic reaction during the study phase but failing to remember anything specific about who said the item (i.e., failing to remember specific source information). The participants were instructed to use a know judgment when they failed to recollect anything specific that would identify who had presented the test item during the study phase. All the participants indicated that they understood the distinction between remember and know responses. All other aspects of the procedure were identical to those in Experiment 1.

\section{Results and Discussion}

Table 3 presents rates of making source attributions of male, female, and new (and the associated responses of
Table 3

Percentages of Responses to Studied and New Words in the Male-Test-Voice, Female-Test-Voice, and No-Test-Voice Conditions in Experiment 2

\begin{tabular}{llrrrrr}
\hline & & \multicolumn{5}{c}{ Response } \\
\cline { 3 - 7 } Test Voice & Source & Male_R & Male_K & Female_R & Female_K & New \\
\hline Male & Male & $\mathbf{3 5 . 3}$ & $\mathbf{3 1 . 2}$ & 4.3 & 8.0 & 21.3 \\
& Female & 11.3 & 19.5 & $\mathbf{2 6 . 9}$ & $\mathbf{1 8 . 0}$ & 24.3 \\
& New & 3.3 & 7.0 & 1.3 & 5.2 & $\mathbf{8 3 . 1}$ \\
Female & Male & $\mathbf{2 2 . 6}$ & $\mathbf{2 3 . 0}$ & 10.2 & 20.4 & 23.7 \\
& Female & 3.2 & 7.1 & $\mathbf{3 9 . 1}$ & $\mathbf{2 7 . 1}$ & 23.6 \\
& New & 1.1 & 3.4 & 2.4 & 8.9 & $\mathbf{8 4 . 2}$ \\
None & Male & $\mathbf{2 6 . 6}$ & $\mathbf{2 3 . 3}$ & 5.6 & 15.3 & 29.2 \\
& Female & 5.2 & 13.6 & $\mathbf{3 3 . 1}$ & $\mathbf{2 1 . 4}$ & 26.8 \\
& New & 2.6 & 3.9 & 1.7 & 6.7 & $\mathbf{8 5 . 1}$ \\
\hline
\end{tabular}

Note-Bold numbers refer to the correct response. R, remember; K, know.

remember and know) to studied items and new items. As for item recognition, a 2 (encoding: male or female study words) $\times 3$ (test voice: male test voice, female test voice, or no test voice) ANOVA of corrected recognition rates revealed no significant effects (all $F_{\mathrm{s}}<1.17$ ). As in Experiment 1, and as shown by Dodson and Shimamura (2000), recognizing items as having been encountered previously was the same in the matching, mismatching, and no-testvoice conditions.

I first assessed overall source identification performance without regard to remember and know judgments by examining the percentage of items recognized as old that were attributed to the correct source. A 2 (encoding) $\times 3$ (test voice) ANOVA of these source scores yielded a significant encoding $\times$ test voice interaction $\left[F(2,52)=26.03, M S_{\mathrm{e}}=0.037, p<.001\right]$ and no other significant effects. Remembering who said something was significantly more accurate in the voice match conditions (i.e., same voices between study and test) than in the voice mismatch conditions (i.e., different voices between study and test) $[t(26)=5.55, p<.001]$. Moreover, as compared with performance in the no-test-voice conditions, source identification performance was significantly better in the voice match conditions $[t(26)=4.95, p<.001]$ and significantly worse in the voice mismatch conditions $[t(26)=4.03, p<.001]$.

I also examined the foregoing data with the identical multinomial model that was used by Dodson and Shimamura (2000, Experiment 1) for a similar data set. This seven-parameter two-high threshold model consists of (1) a parameter, $D$, that measures recognition memory across all of the test conditions; (2) three parameters that measure source memory in the match $\left(d_{\text {match }}\right)$, mismatch $\left(d_{\text {mismatch }}\right)$, and no-test-voice ( $\left.d_{\mathrm{ntv}}\right)$ conditions; (3) two source-guessing bias parameters that measure biases to guess one source or the other in the test voice $\left(a / g_{\text {voices }}\right)$ and no-test-voice $\left(a / g_{\text {ntv }}\right)$ conditions; and (4) a parameter, $b$, that measures the bias to guess that items are old across all conditions (see Dodson \& Shimamura, 2000, for further details about this model). This model fit the data $\left[G^{2}(11)=14.90\right.$, which is less than $G^{2}(11)$ critical $\left.=19.68\right]$.

According to this model, source memory was somewhat better in the match $\left(d_{\text {match }}=.72\right)$ than in the mis- 
match $\left(d_{\text {mismatch }}=.50\right)$ conditions $\left[G^{2}(1)=3.35, p<.07\right]$. Source memory in the no-test-voice condition $\left(d_{\text {neutral }}=\right.$ .57) was not significantly different from performance in either the match or the mismatch condition [both $G^{2}(1) \mathrm{s}<$ 2.64]. Moreover, the participants showed a much stronger source-guessing bias in the test voice conditions $\left(a / g_{\text {voice }}=\right.$ $.66)$ than in the no-test-voice condition $\left(a / g_{\text {neutral }}=.46\right)$ $\left[G^{2}(1)=20.58, p<.0001\right]$. Finally, recognition memory, as indexed by an overall $D$ parameter, was comparable in all the conditions. Overall, then, these model-based analyses replicate what Dodson and Shimamura (2000, Experiment 1) observed and are also consistent with the findings from the foregoing ANOVAs.

Source monitoring and remember/know judgments. I next examined false remember responses in the different test conditions. As is shown in Table 3, these false remember scores are determined by computing the percentage of recognized items given a remember response that were attributed to the wrong source. For instance, false remember responses to items originally presented by the female at encoding would be computed with this formula: $p$ ("Male \& Recollection"|female) $/ p$ ("Male \& Recollection"|female + "Female \& Recollection"|female). A 2 (encoding: male or female) $\times 3$ (test voice: male, female, or none) ANOVA of the false remember responses yielded a significant interaction $\left[F(2,46)=12.19, M S_{\mathrm{e}}=\right.$ $0.044, p<.001]$ and no other significant effects (all $\left.F_{s}<1\right){ }^{2}$ Subsequent planned comparisons showed that there were many more false remember responses in the study-test voice mismatch $(30 \%)$ condition than in the study-test voice match $(9 \%)$ condition $[F(1,46)=24.08$, $p<.001]$. Moreover, there were more false remember responses in the mismatch condition than in the no-test-voice $(15 \%)$ condition $[F(2,46)=7.95, p<.01]$ and fewer false remember responses in the match condition than in the no-test-voice condition $[F(2,46)=4.35, p<.05]$. These results exactly fit the predictions of the illusory recollection account. Mismatching study-test conditions cause an increase in false remember responses.

It is important to note that the foregoing analysis is essentially identical to an analysis of source identification scores that are based on remember responses. The difference is that the foregoing analysis examines incorrect attributions (i.e., false remember responses), whereas a typical analysis of remember source scores examines correct attributions (i.e., the complement of false remember responses).

Finally, I examined source identification performance on the basis of know responses. These know source scores were derived from the percentage of recognized items given a know response that were attributed to the correct source. A 2 (encoding) $\times 3$ (test voice) ANOVA of these source scores revealed a significant test voice $\times$ encoding interaction $\left[F(2,48)=12.33, M S_{\mathrm{e}}=0.061, p<.001\right]$ and no other significant effects (all $F \mathrm{~s}<1) .{ }^{3}$ The know source scores showed the same pattern as the analysis of the overall source scores: Source identification performance was best in the study-test match condition (79\%), worst in the mismatch condition $(51 \%)$, and in-between in the no-testvoice condition $(61 \%)$; all the conditions were significantly different from each other (all $t \mathrm{~s}>2.34, p \mathrm{~s}<.05$ ).
The $\mathbf{R} / \mathrm{K}$ source multinomial model. One problem with current multinomial models is that they confound the influence of guessing and illusory recollection processes, because both processes can contribute to source identification errors. For instance, in the foregoing multinomial model of the overall source scores, the strong source guessing bias $\left(a / g_{\text {voice }}=.66\right)$ in the test voice conditions could be due to the influence of some mixture of guessing and illusory recollection processes. There is no way to disentangle these processes when analyzing overall source attribution scores. However, one advantage of collecting remember/know responses on the source test is that they allow for the separation of guessing processes and illusory recollections.

As is presented in Figure 1, this illusory recollection model assumes that participants recognize items presented at study by the male and female as old and new items as $n e w$ with probabilities $D$ and $D_{\mathrm{n}}$, respectively (Batchelder, Hu, \& Riefer, 1994; Bayen, Murnane, \& Erdfelder, 1996). Building on the model developed by Meiser and Bröder (2002), I included an $R$ parameter to fit the frequency of remember and know responses to items judged old. Following the $D$ branch, the $R$ and $1-R$ branches refer to the probability that source attributions for recognized items are given remember and know responses, respectively. When in a remember state, with a probability of $R$, participants either retrieve diagnostic source information, with a probability of $d R$, and correctly identify the source of the test item or they fail to retrieve diagnostic source information, with a probability of $1-d R$, and experience an illusory recollection for either the male voice or the female voice, with probabilities of $i R e c$ and $1-i R e c$, respectively. When in a know state, with probability $1-R$, participants retrieve diagnostic source information, with a probability of $d K$, and respond with the correct source or they fail to retrieve diagnostic source information, with a probability of $1-d K$, and guess the male source or the female source, with probabilities of $a$ and $1-a$, respectively.

Finally, for studied items and new items that are not recognized as old or new, with probabilities of $1-D$ and $1-$ $D n$, respectively, the $b$ parameter refers to the probability that these items are guessed old. Again, following Meiser and Bröder (2002), the $R^{*}$ and $1-R^{*}$ branches refer to unrecognized targets and new items that are associated with remember and know responses, respectively. The $R^{*}$ branch leads to an illusory recollection for the male source or the female source, with probabilities igRec and $1-i g R e c$, respectively. By contrast, the $1-R^{*}$ (i.e., the know) branch leads to a guessing response for the male source or the female source, with probabilities of $g$ and $1-g$, respectively.

There are two critical assumptions of this model: (1) Source attribution errors that are accompanied by remember responses are due to illusory recollections; (2) source attribution errors that are accompanied by know responses are due to guessing processes. These two assumptions underscore the central point of this article that memory errors can be caused by false recollections as well as by guessing processes, in the absence of retrieval of recollective details. 


\section{Response}
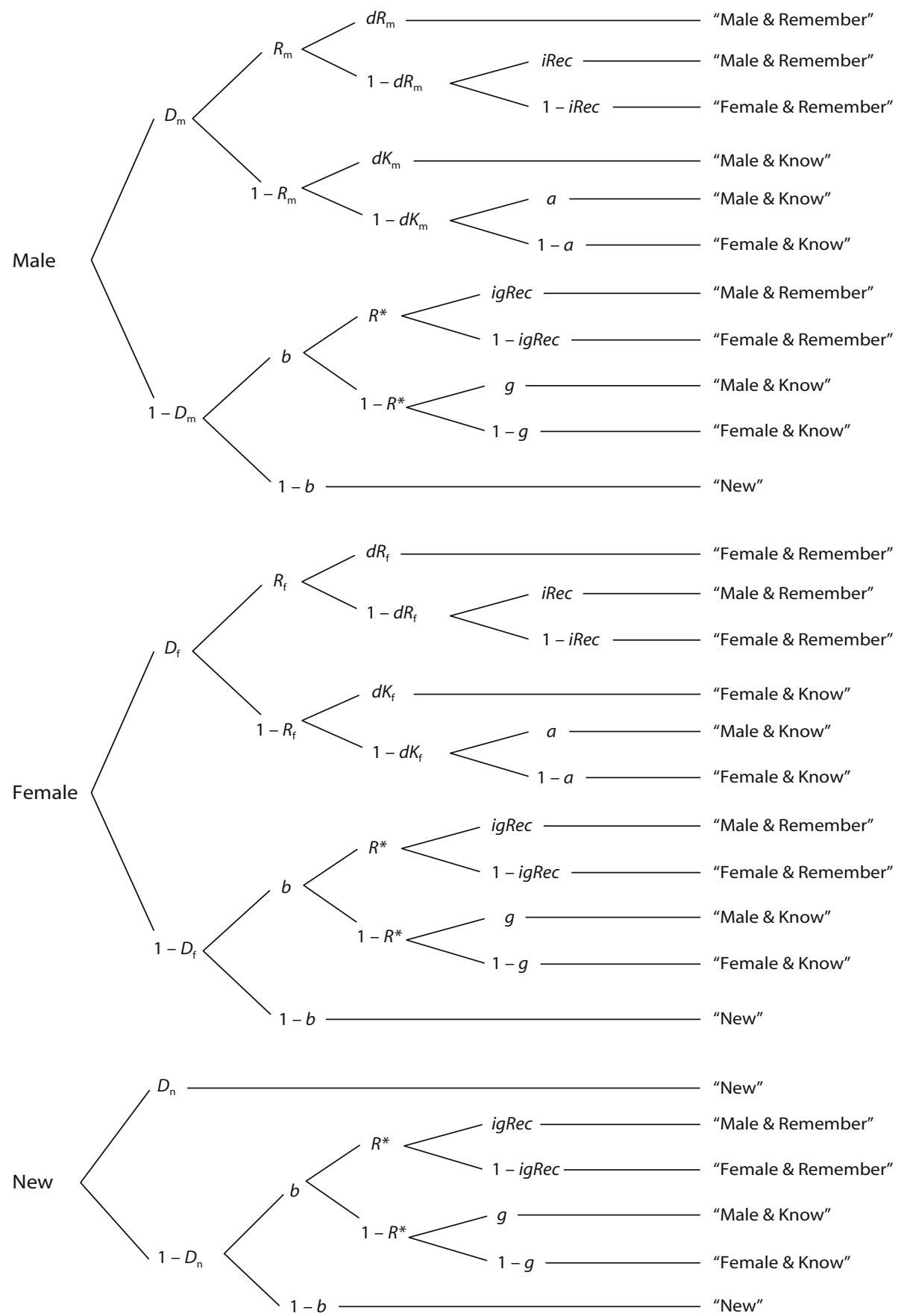

Figure 1. Tree diagrams for the two-high threshold remember/know model of source memory, with separate trees for study items presented by the male and female and for new items on the test. $D_{\mathrm{m}}$, probability of recognizing male study items as old; $D_{\mathrm{f}}$, probability of recognizing female study items as old; $D_{n}$, probability of recognizing new test items as new; $d R_{\mathrm{m}}$, probability of discriminating and remembering the correct source of male study items; $d R_{\mathrm{f}}$, probability of discriminating and remembering the correct source of female study items;

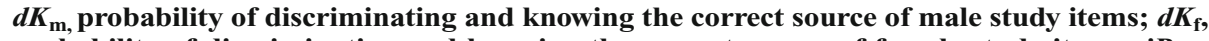
probability of discriminating and knowing the correct source of female study items; $i$ Rec, probability that recognized items are illusorily recollected from the male source; igRec, probability that items that are guessed old are remembered from the male source; $R$, probability of making a remember response to recognized items; $R^{*}$, probability of making a remember response to items that are guessed old; $a$, probability of guessing the male source for recognized items; $g$, probability of guessing the male source for items that are guessed old; $b$, probability of guessing that items are old. 
Table 4

Parameter Values From the Illusory Recollection Model

\begin{tabular}{ccc}
\hline & \multicolumn{2}{c}{ Test Voice Condition } \\
\cline { 2 - 3 } Parameter & Test Voice & No Test Voice \\
\hline$i R e c$ & .76 & .49 \\
$a / g$ & .69 & .47 \\
$d R$ & .68 & .79 \\
$d K$ & .44 & .31 \\
$D$ & .60 & .57 \\
$b$ & .41 & .35 \\
$R_{\mathrm{m}}$ & .52 & .50 \\
$R_{\mathrm{f}}$ & .61 & .59 \\
$R^{*}$ & .25 & .29 \\
\hline
\end{tabular}

Note-iRec, probability of experiencing an illusory recollection; $a / g$, probability of guessing the item was presented by one source or the other; $d R$ and $d K$, source memory for remember responses and know responses, respectively ( 0 equals chance); $D$, item recognition ( 0 equals chance); $b$, bias to recognize an item as old; $R_{\mathrm{m}}$ and $R_{\mathrm{f}}$, probability that source attributions are given remember responses for recognized items that were presented by the male and by the female at encoding, respectively; $R^{*}$, probability that source attributions for unrecognized items are given remember responses. For the test voice condition, $G^{2}(15)=$ $16.19, G^{2}$ critical $=24.99$. For the no-test-voice condition, $G^{2}(3)=5.40$, $G^{2}$ critical $=7.82$.

In sum, as is shown in Figure 1, the illusory recollection model uses 15 parameters to characterize performance on items initially presented by the male $(\mathrm{m})$ source and by the female (f) source at encoding and for new (n) items: $D_{\mathrm{m}}, D_{\mathrm{f}}, D_{\mathrm{n}}, R_{\mathrm{m}}, R_{\mathrm{f}}, R * d R_{\mathrm{m}}, d R_{\mathrm{f}}, d K_{\mathrm{m}}, d K_{\mathrm{f}}, i R e c, i g R e c$, $a, g$, and $b$. To avoid overdetermination of the parameter space, I reduced the number of parameters by equating various parameters within each test voice condition (e.g., Batchelder \& Riefer, 1990; Dodson, Prinzmetal, \& Shimamura, 1998). First, I equated item recognition for the three sources $\left(D_{\mathrm{m}}=D_{\mathrm{f}}=D_{\mathrm{n}}\right)$. Second, I equated the source identification parameters that are accompanied by remember responses $\left(d R_{\mathrm{m}}=d R_{\mathrm{f}}\right)$, and the source identification parameters that are accompanied by know responses $\left(d K_{\mathrm{m}}=d K_{\mathrm{f}}\right)$. Third, I equated the illusory recollection parameters that are associated with items that are recognized as old or guessed old $(i \operatorname{Rec}=i g R e c)$. Finally, I equated the source guessing biases $(a=g)$ that are associated with the bias to guess one source or another for recognized and unrecognized items. In sum, I reduced the model to 9 parameters that have the suffix ntv to refer to the no test voice condition: $D \_n t v, R_{\mathrm{m} \_n t v}, R_{\mathrm{f} \_n t v}, R^{*} \_\mathrm{ntv}$, $d R \_n t v, d K \_n t v, i R e c \_n t v, a / g \_n t v$, and $b \_n t v$. This model fit the data from the no-test-voice condition $\left[G^{2}(3)=5.40\right.$ which is less than $G^{2}(3)$ critical $\left.=7.81\right]$.

For the two test voice conditions, I further simplified this model so that it would fit the combined data sets from the male test voice and female test voice conditions. This was done by equating the following parameters across the test voice conditions: $D$ (item recognition), $b$ (false alarm guessing bias), $d R$ (remember source discrimination), $d K$ (know source discrimination), $R_{\mathrm{m}}$ (probability of being in a remember state to male items), $R_{\mathrm{f}}$ (probability of being in a Remember state to female items), and $R^{*}$ (probability of being in a remember state to new items). In addition, I reformulated the iRec parameter to reflect illusory recollections in terms of the test voice condition. For instance, this parameter reflects the tendency to experience an illusory recollection that a male presented the item in the male test voice condition and that a female presented the item in the female test voice condition. The exact same kind of reformulation occurred for the $a / g$ guessing parameter so that it refers to the probability of guessing in a manner that is consistent with the test voice. Overall, then, the model for the test voice conditions contained 9 parameters: $D \_t v, R_{\mathrm{m} \_t}$, $R_{\mathrm{f} \_} \mathrm{tv}, R^{*} \_\mathrm{tv}, d \mathrm{R} \_\mathrm{tv}, d \mathrm{~K} \_\mathrm{tv}, i R e c \_\mathrm{tv}, a / g \_\mathrm{tv}$, and $b \_\mathrm{tv}$. This test voice model provided a good fit to the data $\left[\bar{G}^{2}(15)=\right.$ 16.19 is less than $G^{2}(15)$ critical $\left.=24.99\right]$.

$\mathbf{R} / \mathbf{K}$ source model analyses. Table 4 shows the parameter values for the test voice and no-test-voice conditions. I first analyzed the illusory recollection (iRec) and source guessing $(\mathrm{a} / \mathrm{g})$ parameters. For each of these parameters, values of .5 mean that there is an equal tendency to experience illusory recollections for or to guess one source or the other. Values greater than or less than .5 mean that there is an asymmetry toward experiencing an illusory recollection for or preferentially guessing one source over the other.

As can be seen in Table 4, illusory recollections were strongly influenced by the presence of test voices. For instance, for the no-test-voice condition the value of $i R e c$ (.49) was nearly identical to .5 , indicating that when illusory recollections occurred, they were nearly equally likely to occur for the male and the female sources. However, the value of $i R e c$ for the test voices condition (.76) was significantly larger than the value of $i R e c$ for the notest-voice condition (.49) $\left[G^{2}(1)=20.41, p<.001\right]$. In short, when the participants were in a remember state and failed to retrieve diagnostic source information, they were likely to experience an illusory recollection that was consistent with the test voice $76 \%$ of the time.

At the same time, test voices also influenced guessing biases for one source over the other. The value of the $\mathrm{a} / \mathrm{g}$ guessing parameter was significantly larger in the test voice conditions (.69) than in the no-test-voice condition $(.47)\left[G^{2}(1)=41.89, p<.001\right]$. In fact, the participants in the no-test-voice condition showed almost no bias to preferentially guess one source or the other, since .47 is not significantly different from .5 [i.e., the value corresponding to no bias; $\left.G^{2}(1)=1.23, p>.25\right]$.

I next examined the relative magnitude of the influence of test voices on illusory recollections and on guessing biases by comparing the values of the illusory recollection (iRec) and source-guessing $(a)$ parameters. For the test voice conditions, there was a somewhat higher probability of experiencing illusory recollections in a test-voice-consistent manner $(i R e c=.76)$ than there was of guessing in a testvoice-consistent manner $(a=.69)\left[G^{2}(1)=3.50, p=.06\right]$ For the no-test-voice condition, both illusory recollections $(i \operatorname{Rec}=.49)$ and guessing biases $(a / g=.47)$ were not significantly different from each other $\left[G^{2}(1)<.5\right]$. Providing test voices at retrieval has a somewhat larger influence on the creation of illusory recollections than it does on influencing guessing biases.

The remaining question of interest concerns the influence of test voices on the source discrimination parameters that measure source memory after factoring out the influence of illusory recollections and guessing biases. As can 
be seen in Table 4, in both the test voice and the no-testvoice conditions, source memory was better when it was accompanied by remember responses (i.e., $d$ _Remember) than by know responses (i.e., $d \_$Know) [both $G^{2}(1) \mathrm{s}>$ $15.03, p \mathrm{~s}<.01]$. However, within the test voice conditions, source memory-regardless of whether it was accompanied by remember or know responses-was not significantly different when there was either a matching or a mismatching test voice [both $G^{2}(1) \mathrm{s}<1.28, p \mathrm{~s}>.25$ ]. That is, including separate source memory parameters for the matching and mismatching test voice conditions that are accompanied by either remember responses (i.e., $d \mathrm{R} \_$match and $d \mathrm{R} \_$mismatch) or know responses (i.e., $d \mathrm{~K} \_$match and $d \mathrm{~K} \_$mismatch) did not improve the fit of the model. This means that matching and mismatching test voices primarily influence memory performance by influencing illusory recollections and guessing biases. Once these processes are factored out of performance, there is no significant difference between matching and mismatching test voices on the source discrimination parameters.

However, as can be seen in Table 4, the source memory parameter was better in the no-test-voice condition (.79) than in the test voice condition (.68) when source judgments were accompanied by remember responses $\left[G^{2}(1)=3.61, p=.06\right]$. Thus, this result indicates that test voices impair recollective-based source discrimination because of the occurrence of illusory recollections. I will discuss this result further in the General Discussion section. Source discrimination that was accompanied by know responses was not significantly different in the test voice and the no-test-voice conditions $\left[G^{2}(1)=2.19\right]$.

As for the remaining parameters, item recognition (D) was no different between the test voice (.60) and the notest-voice $(.57)$ conditions $\left[G^{2}(1)=1.75\right]$. However, the participants tended to show a more conservative false alarm guessing bias in the no-test-voice condition than in the test voice conditions $\left[b=.35\right.$ vs. .41 , respectively; $G^{2}(1)=$ $4.17, p<.05]$. Finally, there were no differences between the test voice and no test voice conditions in the likelihood of being in a remember state either for the items presented by the male and female at encoding or for the new items [i.e., the $R \mathrm{~m}, R_{\mathrm{f}}$, and $R^{*}$ parameters; all $G^{2}(1) \mathrm{s}<1$ ].

\section{GENERAL DISCUSSION}

It is well established that memory performance is influenced by the degree of match between the conditions at encoding and at retrieval. Both experiments show that remembering who said something earlier was superior when the test voice matched, rather than mismatched, the study voice. I examined two different hypotheses - the illusory recollection and the recollection failure accountsfor why source identification performance is worse when the retrieval context mismatches, rather than matches, the properties of the memory trace. The results from both experiments support an illusory recollection account. Mismatching test voices cause errors by creating illusory recollections that are consistent with the test voice.

In Experiment 1, the mismatching retrieval context did not interfere with or block access to the desired source memory, which would have led to an increase in don't remember responses in the mismatching test voices condition. In fact, the overall rate of responding don't remember was nearly identical when test voices either matched or mismatched the actual speaker of the item, despite the fact that overall memory performance was much worse in the mismatching condition. Instead, mismatching test voices caused frequent selection of the wrong source. Experiment 2 used a remember/know procedure and obtained similar results. Mismatching test voice conditions increased the likelihood of false remember responses whereby the participants chose the wrong source apparently because they falsely remembered specific details about this source's presenting the test item during the encoding phase. Moreover, the remember/know procedure allowed for the use of a multinomial model that was able to separate and measure the influence of test voices on illusory recollections and source discrimination. This model further indicated that test voices manufacture illusory recollections.

By illusory recollection, I refer to experiences that are shaped primarily by the properties of the retrieval environment. In other words, the retrieval conditions "capture" recollective experience - to use Jacoby and colleagues' term-so that individuals believe that they are recollecting a past event when in fact they are misattributing features of the test voice (Jacoby, Bishara, Hessels, \& Toth, 2005). One implication of this that is instantiated in the multinomial model is that illusory recollections will not always lead to incorrect responses. If test voices primarily determine recollective experience, this recollective experience will lead to correct responses in the study-test match conditions and incorrect responses in the studytest mismatch conditions. Even though they may lead to a correct response, these recollections are illusory because they have been captured by - they are derived primarily from - the test voice and are not fueled by what was encoded. Consequently, it is critical to try to separate the influence of illusory recollections from source memory, since under certain circumstances both processes can lead to correct responses. This is the goal of the illusory recollection multinomial model.

The multinomial model indicates that once illusory recollections and guessing biases have been factored out of performance overall source memory, as measured by the source discrimination parameters, is no different in the study-test match and mismatch conditions. This lack of difference in the source parameters does not contradict the behavioral result that source identification performance is worse in the mismatch than in the match conditions. What the modeling results mean is that this difference in source identification performance is substantially caused by the influence of illusory recollections.

It is important to note that guessing does occur in this paradigm. For instance, in Experiment 1, the participants responded don't remember nearly $30 \%$ of the time when they likely would have guessed a response if not for this response option. Moreover, Experiment 2 (which did not have a don't remember option) shows that the participants were strongly biased to guess in a manner that was consis- 
tent with the test voice. However, one of the key points of these experiments is that guessing by itself cannot completely explain the effect of matching and mismatching test conditions on participants' ability to remember who said something.

I suggest that the cause of illusory source recollections follows from Tulving's (1983) theory of synergistic ecphory. Synergistic ecphory refers to the notion that recollective experience does not just mirror the activated properties of the memory trace. Instead, recollections are constructed both from the trace information and from the information in the retrieval environment - an assumption that is a basic tenet of the source-monitoring framework of Johnson and colleagues and the constructive memory framework of Schacter and colleagues (Johnson et al., 1993; Schacter et al., 1998). Illusory recollections about a particular source, therefore, may occur when misleading information in the retrieval cue, such as in the mismatch test voice condition, is incorporated into the recollective experience.

There is some degree of similarity between the notion of retrieval-provoked illusory recollections and Jacoby and colleagues' notion that retrieval cues can capture behavior and lead to instances of false remembering (Jacoby et al., 2005). Jacoby et al. showed that during a cued recall test when items were briefly preceded by a prime word (e.g., bend) that either matched or mismatched the answer to a test item (e.g., knee $\left.-b \_n \_?\right)$, participants, and particularly older adults, tended to accept the prime word as a valid response and even to report that they recollected studying the word earlier when, in fact, it was misleading and had not been encountered in the experiment. To explain these instances of false remembering, Jacoby et al. proposed that individuals are prone to being captured by the prime word so that they fail to carry out the process of recollection. Although there are substantial differences in my and Jacoby and colleagues' multinomial models about where the illusory recollection (i.e., the "capture") process fits in with the other memorial processes, both accounts highlight the importance of retrieval conditions as a cause of false recollective experiences.

In sum, contextual changes between the encoding and the retrieval environments hurt performance because participants are likely to experience illusory recollections that are consistent with the retrieval context. The theory of retrieval-based illusory recollections suggests that any information in the retrieval cue, not merely perceptual voice information, can give rise to illusory recollections. In support of this, I have shown that when conceptual stereotype information is introduced during a memory test, this information creates illusory source recollections that are consistent with the stereotype (Dodson, Darragh, \& Williams, in press). Various factors at retrieval - the context in the environment or one's expectations about what should be remembered - can cause false recollections that are consistent with these factors.

\section{AUTHOR NOTE}

Correspondence concerning this article should be addressed to C. S. Dodson, Department of Psychology, University of Virginia, P.O. Box
400400, 102 Gilmer Hall, Charlottesville, VA 22904-4400 (e-mail: cdodson@virginia.edu).

\section{REFERENCES}

ANDERSON, J. R. (1983). A spreading activation theory of memory. Journal of Verbal Learning \& Verbal Behavior, 22, 261-295.

ANDERSON, M. C., \& NeELy, J. H. (1996). Interference and inhibition in memory retrieval. In E. A. Bjork \& R. A. Bjork (Eds.), Memory (pp. 237-313). San Diego: Academic Press.

Batchelder, W. H., Hu, X., \& Riefer, D. M. (1994). Analysis of a model for source monitoring. In G. H. Fischer \& D. Laming (Eds.), Contributions to mathematical psychology, psychometrics, and methodology (pp. 51-65). New York: Springer.

BAtchelder, W. H., \& Riefer, D. M. (1990). Multinomial processing models of source monitoring. Psychological Review, 97, 548-564.

Batchelder, W. H., \& Riefer, D. M. (1999). Theoretical and empirical review of multinomial process tree modeling. Psychonomic Bulletin \& Review, 6, 57-86.

Bayen, U. J., Murnane, K., \& Erdfelder, E. (1996). Source discrimination, item detection, and multinomial models of source monitoring. Journal of Experimental Psychology: Learning, Memory, \& Cognition, 22, 197-215.

Bower, G. H. (1967). A multi-component theory of the memory trace. In K. W. Spence \& J. T. Spence (Eds.), The psychology of learning and motivation: Advances in research and theory (Vol. 1, pp. 299-325). New York: Academic Press.

Brainerd, C. J., Payne, D. G., Wright, R., \& Reyna, V. F. (2003). Phantom recall. Journal of Memory \& Language, 48, 445-467.

Dewhurst, S. A., \& Hitch, G. J. (1999). Cognitive effort and recollective experience in recognition memory. Memory, 7, 129-146.

Dodson, C. S., Darragh, J., \& Williams, A. (in press). Stereotypes and retrieval-provoked illusory source recollections. Journal of Experimental Psychology: Learning, Memory, \& Cognition.

Dodson, C. S., \& Krueger, L. E. (2006). I misremember it well: Why older adults are unreliable eyewitnesses. Psychonomic Bulletin \& Review, 13, 770-775.

Dodson, C. S., Prinzmetal, W., \& Shimamura, A. P. (1998). Using Excel to estimate parameters from observed data: An example from source memory data. Behavior Research Methods, Instruments, \& Computers, 30, 517-526.

Dodson, C. S., \& Shimamura, A. P. (2000). Differential effects of cue dependency for item and source memory. Journal of Experimental Psychology: Learning, Memory, \& Cognition, 26, 1023-1044.

Frost, P. (2000). The quality of false memory over time: Is memory for misinformation "remembered" or "known"? Psychonomic Bulletin \& Review, 7, 531-536.

Gallo, D. A., \& Roediger, H. L., III (2003). The effects of associations and aging on illusory recollection. Memory \& Cognition, 31, 1036-1044.

GARDINER, J. M., \& JAVA, R. I. (1993). Recognizing and remembering. In A. F. Collins, S. E. Gathercole, M. A. Conway, \& P. E. Morris (Eds.), Theories of memory (pp. 163-188). Hove, U.K.: Erlbaum.

Godden, D. R., \& Baddeley, A. D. (1975). Context dependency in two natural environments: On land and underwater. British Journal of Psychology, 66, 325-331.

Hannigan, S. L., \& Reinitz, M. T. (2001). A demonstration and comparison of two types of inference-based memory errors. Journal of Experimental Psychology: Learning, Memory, \& Cognition, 27, 931940

Hicks, J. L., Marsh, R. L., \& Ritschel, L. (2002). The role of recollection and partial information in source monitoring. Journal of Experimental Psychology: Learning, Memory, \& Cognition, 28, 503-508.

Higham, P. A., \& VoKey, J. R. (2004). Illusory recollection and dualprocess models of recognition memory. Quarterly Journal of Experimental Psychology, 57A, 714-744.

HintzMan, D. L. (1986). "Schema abstraction" in a multiple-trace memory model. Psychological Review, 93, 411-428.

Jacoby, L. L., Bishara, A. J., Hessels, S., \& Toth, J. P. (2005). Aging, subjective experience, and cognitive control: Dramatic false remembering by older adults. Journal of Experimental Psychology: General, 134, 131-148. 
Johnson, M. K., Hashtroudi, S., \& Lindsay, D. S. (1993). Source monitoring. Psychological Bulletin, 114, 3-28.

KuČERA, H., \& Francis, W. N. (1982). Frequency analysis of English usage: Lexicon and grammar. Boston: Houghton Mifflin.

Lampinen, J. M., Meier, C. R., Arnal, J. D., \& Leding, J. K. (2005). Compelling untruths: Content borrowing and vivid false memories. Journal of Experimental Psychology: Learning, Memory, \& Cognition, 31, 954-963.

Lampinen, J. M., Neuschatz, J. S., \& Payne, D. G. (1999). Source attributions and false memories: A test of the demand characteristics account. Psychonomic Bulletin \& Review, 6, 130-135.

Leynes, P. A., Bink, M. L., Marsh, R. L., Allen, J. D., \& May, J. C. (2003). Test modality affects source monitoring and event-related potentials. American Journal of Psychology, 116, 389-413.

LiNDSAY, D. S. (1990). Misleading suggestions can impair eyewitnesses' ability to remember event details. Journal of Experimental Psychology: Learning, Memory, \& Cognition, 16, 1077-1083.

LindSAY, D. S., \& Kelley, C. M. (1996). Creating illusions of familiarity in a cued recall remember/know paradigm. Journal of Memory \& Language, 35, 197-211.

Macrae, C. N., Schloerscheidt, A. M., Bodenhausen, G. V., \& Milne, A. B. (2002). Creating memory illusions: Expectancy-based processing and the generation of false memories. Memory, 10, 63-80.

McGeoch, J. A. (1942). The psychology of human learning: An introduction. New York: Longmans Green.

MeISER, T., \& BRÖDER, A. (2002). Memory for multidimensional source information. Journal of Experimental Psychology: Learning, Memory, \& Cognition, 28, 116-137.

Morris, C. D., Bransford, J. D., \& Franks, J. J. (1977). Levels of processing versus transfer appropriate processing. Journal of Verbal Learning \& Verbal Behavior, 16, 519-533.

Murnane, K., \& Bayen, U. J. (1996). An evaluation of empirical measures of source identification. Memory \& Cognition, 24, 417-428.

Murnane, K., Phelps, M. P., \& Malmberg, K. (1999). Contextdependent recognition memory: The ICE theory. Journal of Experimental Psychology: General, 128, 1-13.

NAIRne, J. S. (2002). The myth of the encoding-retrieval match. Memory, 10, 389-395.

Ochsner, K. N., Schacter, D. L., \& Edwards, K. (1997). Illusory recall of vocal affect. Memory, 5, 433-455.

Palmeri, T. J., Goldinger, S. D., \& Pisoni, D. B. (1993). Episodic encoding of voice attributes and recognition memory for spoken words. Journal of Experimental Psychology: Learning, Memory, \& Cognition, 19, 309-328.

Payne, D. G., Elie, C. J., Blackwell, J. M., \& Neuschatz, J. S. (1996). Memory illusions: Recalling, recognizing, and recollecting events that never occurred. Journal of Memory \& Language, 35, 261285.

Perfect, T. J., Mayes, A. R., Downes, J. J., \& Van EiJK, R. (1996). Does context discriminate recollection from familiarity in recognition memory? Quarterly Journal of Experimental Psychology: Human Experimental Psychology, 49A, 797-813.

RaAijmakers, J. G., \& Shiffrin, R. M. (1981). Search of associative memory. Psychological Review, 88, 93-134.
Rajaram, S. (1993). Remembering and knowing: Two means of access to the personal past. Memory \& Cognition, 21, 89-102.

Roediger, H. L., III, McDermott, K. B., Pisoni, D. B., \& Gallo, D. A. (2004). Illusory recollection of voices. Memory, 12, 586-602.

Roediger, H. L., III, Weldon, M. S., \& Challis, B. H. (1989). Explaining dissociations between implicit and explicit measures of retention: A processing account. In H. L. Roediger III \& F. I. M. Craik (Eds.), Varieties of memory and consciousness: Essays in honour of Endel Tulving (pp. 3-41). Hillsdale, NJ: Erlbaum.

SCHACTER, D. L. (2001). Forgotten ideas, neglected pioneers: Richard Semon and the story of memory. New York: Psychology Press.

Schacter, D. L., Norman, K. A., \& Koutstaal, W. (1998). The cognitive neurosciences of constructive memory. Annual Review of Psychology, 49, 289-318.

Schacter, D. L., \& Slotnick, S. D. (2004). The cognitive neuroscience of memory distortion. Neuron, 44, 149-160.

Senkfor, A. J., \& Van Petten, C. (1998). Who said what? An eventrelated potential investigation of source and item memory. Journal of Experimental Psychology: Learning, Memory, \& Cognition, 24, $1005-1025$

Smith, S. M., \& Vela, E. (2001). Environmental context-dependent memory: A review and meta-analysis. Psychonomic Bulletin \& Review, 8, 203-220.

SoundEDIT [Computer software] (1992). San Francisco: Macromedia.

Tulving, E. (1983). Elements of episodic memory. Oxford: Oxford University Press, Clarendon Press.

Tulving, E. (1985). Memory and consciousness. Canadian Psychologist, 26, 1-12.

Tulving, E., \& Thomson, D. M. (1973). Encoding specificity and retrieval processes in episodic memory. Psychological Review, 80, 352-373.

Whittlesea, B. W. A. (2004). The perception of integrality: Remembering through the validation of expectation. Journal of Experimental Psychology: Learning, Memory, \& Cognition, 30, 891-908.

WiXTED, J. T. (2004). The psychology and neuroscience of forgetting. Annual Review of Psychology, 55, 235-269.

Zaragoza, M. S., \& LANE, S. M. (1994). Source misattributions and the suggestibility of eyewitness memory. Journal of Experimental Psychology: Learning, Memory, \& Cognition, 20, 934-945.

\section{NOTES}

1. After completing the source test, the participants completed an additional source memory test in which all of the test words were presented visually only; no test item was presented by a voice. However, due to space constraints, the results from this second test will not be discussed.

2. The degrees of freedom are reduced because 3 participants did not use the remember response to a particular item type in one of the test voice conditions.

3. The degrees of freedom are reduced because 2 participants did not use the know response to a particular item type in one of the test voice conditions.

(Manuscript received March 4, 2005; revision accepted for publication July 4, 2006.) 